Article

\title{
Silver Nanoparticles Ecofriendly Synthesized by Achyranthes aspera and Scoparia dulcis Leaf Broth as an Effective Fungicide
}

\author{
Ngoc Thuy Trang Le ${ }^{1}\left(\mathbb{D}\right.$, Dai Hai Nguyen ${ }^{2,3}{ }^{\mathbb{D}}$, Ngoc Hoi Nguyen ${ }^{2}$, Yern Chee Ching ${ }^{4}$, \\ Dong Yen Pham Nguyen ${ }^{2}$, Cuong Quoc Ngo ${ }^{5}$, Hang Nguyen Thi Nhat ${ }^{6}$ and \\ Thai Thanh Hoang Thi ${ }^{5, * \mathbb{D}}$ \\ 1 Institute of Research and Development, Duy Tan University, Danang 550000, Vietnam; \\ lenthuytrang4@duytan.edu.vn \\ 2 Institute of Applied Materials Science, Vietnam Academy of Science and Technology, 01 TL29 District 12, \\ Ho Chi Minh City 700000, Vietnam; nguyendaihai0511@gmail.com (D.H.N.); hoi83bmt@gmail.com (N.H.N.); \\ phamngdongyen@gmail.com (D.Y.P.N.) \\ 3 Graduate University of Science and Technology, Vietnam Academy of Science and Technology, Hanoi 100000, \\ Vietnam \\ 4 Department of Chemical Engineering, Faculty of Engineering, University of Malaya, Kuala Lumpur 50603, \\ Malaysia; chingyc@um.edu.my \\ 5 Biomaterials and Nanotechnology Research Group, Faculty of Applied Sciences, Ton Duc Thang University, \\ Ho Chi Minh City 700000, Vietnam; 186002002@student.tdtu.edu.vn \\ 6 Faculty of Natural Sciences, Thu Dau Mot University, Thu Dau Mot City 820000, Binh Duong Province, \\ Vietnam; hangntn@tdmu.edu.vn \\ * Correspondence: hoangthithaithanh@tdtu.edu.vn; Tel.: +84-(0)28-3775-5058
}

Received: 26 February 2020; Accepted: 2 April 2020; Published: 5 April 2020

\begin{abstract}
This study describes an inexpensive, simple and green method to form silver nanoparticles from different leaf extracts of Achyranthes aspera and Scoparia dulcis plants. The silver nitrate is reduced by Achyranthes aspera and Scoparia dulcis leaf extracts respectively to generate two silver nanoparticle types symbolized as AA.AgNPs and SD.AgNPs. The optical absorption, size and morphology of silver nanoparticles are significantly impacted by extract types. The ultraviolet visible spectrum of AA.AgNPs shows a 433-nm peak being more broadened than that of SD.AgNPs. The Fourier infrared transform spectra of two of these silver nanoparticles revealed that their surface is modified by organic constituents from extracts, and thus they are stabilized in solution without any additional reaction. Images from transmission electron microscopy and scanning electron microscope indicate that AA.AgNPs are in clusters with the size of $8-52 \mathrm{~nm}$ almost possessing oval shape, while SD.AgNPs are smaller size of 5-45 nm separated well in diversified shapes (spherical, triangle, quadrilateral and hexagonal). Moreover, both AA.AgNPs and SD.AgNPs exhibit the highly antifungal effect against Aspergillus niger, Aspergillus flavus and the most strong impact on Fusarium oxysporum. For these obtained results, two new silver nanoparticles are promising fungicides for various applications of medical and agricultural fields.
\end{abstract}

Keywords: silver nanoparticle; fungicide; plant extract; Achyranthes aspera; Scoparia dulcis

\section{Introduction}

For a long time, the irregular use of fungicides not only threatened plants, animals and human health but also led to the extreme consequence of antifungal resistance. This problem has been becoming more seriously, the mortality rates related to fungal infection, especially antifungal resistance 
infection, were over 50\% [1]. The epidemiology research revealed that the patient who has not been treated by antifungal-drugs could become antifungal resistance if they inhaled the agricultural fungal spores exposed to fungicide [1]. Besides, the human might contact the azole derivatives, which is one of antifungicides during cultivating and farming, or consume the agricultural products having antifungicide residues [1]. Therefore, in the battle of antifungal resistance [2], the chemical control in crops is necessary [3], and the new antifungal strategy needs to be studied [2,4]. Antimicrobial peptides have been attracted due to a broad spectrum of ability, less susceptible to resistant species and low toxicity, but high cost and short half-life led them to be of interest $[5,6]$. The sequence-controlled copolymers were also designed to gain the great antimicrobial activities [7], but the complicated synthesis and high cost limited them in various applications. Nitric oxide is also the most outstanding molecular in antimicrobial applications, but it is extremely susceptive that leads to many difficulties in synthesis, application and storage [8]. The biosynthesized nanoparticles were considered as the better promising alternative due to adapting many requirements such as cost effects, positively environmental impacts and socially sustainable $[9,10]$. The biosynthesized nanoparticles are formed by an ecofriendly technique or green chemistry. This method utilized less energy, minimized the toxic chemicals, simplified the procedure and exploited the natural materials being able to regenerate [11]. Therefore, the green synthesis of nanoparticles has been more attractive than the physical and chemical methods. Recently, Oh et al. prepared the chitosan nanoparticles (CNPs) through ionic gelation. These obtained CNPs exhibited the highly antifungal ability against Fusarium oxysporum and Phytophthora capsici, and the good antibacterial effects against Xanthomonas and Erwinia strains [12]. The polymeric nanoparticles achieved a great biocompatibility and antifungal ability, but the degradation time was limited. The polymeric nanoparticles might be degraded completely before killing all pathogens.

Other green methods were also studied to utilize the microorganisms to fabricate the metallic nanoparticles. Hassan et al. used Aspergillus niger producing extracellularly silver nanoparticles (AgNPs) [13]. These AgNPs showed good antifungal activities. By similar methods, Iravani summarized a lot of metallic nanoparticles (silver, gold, palladium and selenium, magnetite, cadmium sulphide, lanthanum, zinc sulfide, platinum, etc.) formed by various bacteria (Bacillus cereus, Bacillus subtilis 168, Desulfovibrio desulfuricans, Desulfovibrio magneticus strain RS-1, Escherichia coli, Pseudomonas aeruginosa, Rhodobacter sphaeroides, Shewanella algae, etc.) [14]. The mechanism forming metallic nanoparticles by bacteria was explained by the secreted proteins with reducing property that could oxidize the metallic ions [14]. However, the bacterial culture and the extraction/purification of nanoparticles were complicated, the proteins and enzymes capping nanoparticles have not known their functions or impacts yet. These matters might hurdle the development of microbial nanoparticles. Another popularly green source for nanoparticles biosynthesis was plant extracts [15,16]. The ketones, flavones, carboxylic acids, terpenoids, polyphenols, vitamin $C$ and aldehydes being abundantly in plant extracts were exploited as the reducing agents to oxidize metallic ions and form metallic nanoparticles. This route was considered as a more simple and easier procedure, being able to scale up more than other aforementioned green methods [17]. Although there were many studies that reported the plant extract-mediated nanoparticles, the diversified plant species were still not exploited completely. Depending on the plant extract types, the size, morphology and stabilizing ability of nanoparticles were different, which might affect the activity of formed nanoparticles. Thus the studies related to specific plants or plant parts using for nanoparticle formation still need to be investigated.

Achyranthes aspera Linn. (A. aspera) belongs to the Amaranthaceae family and is well-known as a traditional cure in fold medicine. It had many medicinal functions such as anticancer, antimicrobial, diuretic, antiobesity, antineoplastic, antiherpes, hepatoprotective ability, antineoplastic and immunomodulatory activities, antioxidant and neuroprotective property [18]. Besides, the essential oils of $A$. aspera were demonstrated about an effective protection from two mosquito species including C. quinquefasciatus and Z. officinalis [19]. Aqueous extract of aerial parts containing oleanolic acid could stabilize mast cells contributing helpfully in asthma treatment [20]. A. aspera leaves were proved to enhance the wound healing rate on rat models [21]. In phytochemistry research, $A$. aspera was reported 
to contain alkaloids, flavonoids, betaine derivatives, proteins, amino acids, oligosaccharides, sugars, saponins, steroids and terpenoids [22]. So, there were a lot of studies using A. aspera with various aspects, but the use of its leaf broth to synthesize nanoparticles was only in a few studies. With an attempt to explore the green silver nanoparticles from various plant species, we also utilized other plants of Scoparia dulcis (S. dulcis) to make the comparison. S. dulcis belongs to the Scrophulariaceae family. In folk medicine, it was well-known as a perennial herb with abundant bioactive compounds. Its medicinal activities exhibited on treatment of cancer, diarrhea, diabetes, stomachache, hepatosis, bronchitis, fever, hypertension and ulcers [23]. The phytoconstituents of S. dulcis were abundantly found, those were diterpenoid, triterpenoid, alkaloids, flavonoids and steroids [23,24].

Using the plant extracts to biosynthesize nanoparticles, many studies about Cannabis sativa, Azadirachta indica, Jatropha curcas, etc. were conducted [25-28]. So far, there were no reports on A. aspera or S. dulcis leaf extract-mediated silver nanoparticles on antifungal activity against Aspergillus niger (A. niger), Fusarium oxysporum (F. oxysporum) and Aspergillus flavus (A. flavus). A. niger belongs to the genus Aspergillus, and can cause black mold disease on certain vegetables and fruits. It is commonly found on dead leaves, compost piles, stored grain and other decaying vegetation [29]. A. flavus is of the same genus of $A$. niger, well-known for colonizing on grains, nuts and legumes during postharvest stages. More dangerously, A. flavus could cause human infection [30]. F. oxysporum can destroy the plants gradually by clearing veins, yellowing leaves, stunting trees, etc. [31]. Thus, fungicides are necessary for crops to protect agriculture from being destroyed, more importantly to prevent the fungi infecting humans. However, antifungal resistance occurred, which induced the development of an alternative fungicide. Herein, two kinds of biosynthesized silver nanoparticles would be a good candidate. AgNPs would be fabricated using A. aspera or S. dulcis leaf extracts. The ultraviolet visible spectrophotometer (UV-vis) was carried out to realize the nanoparticle formation. The Fourier transform infrared spectrometer (FTIR) and energy dispersive X-ray analysis (EDXA) were used to show the functional groups and the elements of these AgNPs. Scanning electron microscopy (SEM) and transmission electron microscopy (TEM) were performed to observe the morphology and to estimate the dimension of nanoparticles. Finally, the two types of AgNPs would be mixed with culturing media to grow fungi (A. niger, F. oxysporum and A. flavus) until after $96 \mathrm{~h}$. Measuring the mycelium diameters was done to recognize the inhibitory ability of each AgNP. Through all of the results, we could make a comparison between two AgNPs of A. aspera or S. dulcis leaf extracts as well as comprehending two of these green sources for silver nanoparticle synthesis with specific properties.

\section{Materials and Methods}

\subsection{Materials}

Silver nitrate (ACS reagent, $\geq 99.0 \%$ of purity) and potassium bromide (KBr, FT-IR grade, $\geq 99.0 \%$ trace metals basis) were purchased from Sigma-Aldrich (Merck KGaA, Darmstadt, Germany). Potato dextrose agar (PDA) containing $4 \mathrm{~g} / \mathrm{L}$ of potato extract, $20 \mathrm{~g} / \mathrm{L}$ of dextrose and $15 \mathrm{~g} / \mathrm{L}$ agar was readily mixed in powder, obtained from Sigma-Aldrich (Millipore Sigma, Burlington, Massachusetts, US). All of the experiments were used with deionized water (DIW) filtrated by water purification systems (Milli-Q HX 7150, Merck Millipore, Guyancourt, France).

A. aspera or S. dulcis leaves were harvested from the Medicinal Plant Garden of Tra Vinh University (Tra Vinh province, Vietnam). A. niger, F. oxysporum and A. flavus were isolated by the Institute of Applied Materials Science of Vietnam Academy of Science and Technology (Ho Chi Minh City, Vietnam).

\subsection{Biosynthesis of Silver Nanoparticles Using the A. aspera and S. dulcis Leaf Extracts}

The fresh A. aspera and S. dulcis leaves without defects and diseases were carefully collected and put into different baskets. Two kinds of plant leaves were washed three times to eliminate all dust. Each leaf type was chopped, and then weighed $5 \mathrm{~g}$ into each Erlenmeyer flask (Figure 1). Of DIW, 
$50 \mathrm{~mL}$ was poured into each flask. Two of these Erlenmeyer flasks were heated up to $60{ }^{\circ} \mathrm{C}$ while stirring continuously. This process was kept for $60 \mathrm{~min}$. After heating, the filtration was carried out with a Buchner filtration system using Whatman ${ }^{\circledR}$ quantitative filter paper (hardened low ash, grade 50, Merck KGaA, Darmstadt, Germany). The aqueous extract of $A$. aspera or $S$. dulcis leaves symbolized AA.ext and SD.ext respectively and were stored at $4{ }^{\circ} \mathrm{C}$ for 7 days for further experiments.

Silver nitrate was prepared in DIW at a concentration of $1 \mathrm{mM}$. The Erlenmeyer flask contained $8 \mathrm{~mL}$ of AA.ext broth. A volume of $1.2 \mathrm{~mL}$ of silver nitrate solution was pipetted, and then was dropped into the AA.ext broth while stirring continuously (Figure 1). After adding silver nitrate completely, the reaction was kept for $8 \mathrm{~h}$ at room temperature in a dark place. The silver nanoparticles (AA.AgNPs) formed by the AA.ext broth were purified with DIW three times. The centrifuge was used to collect the AgNP suspension. They were lyophilized and stored under vacuum. Proceeding similarly to the AA.AgNP synthesis procedure, the SD.ext was utilized instead of AA.ext, the SD.ext broth-mediated silver nanoparticles (SD.AgNPs) were obtained.

(a)

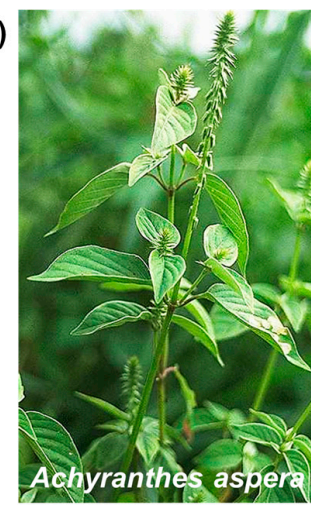

(b)
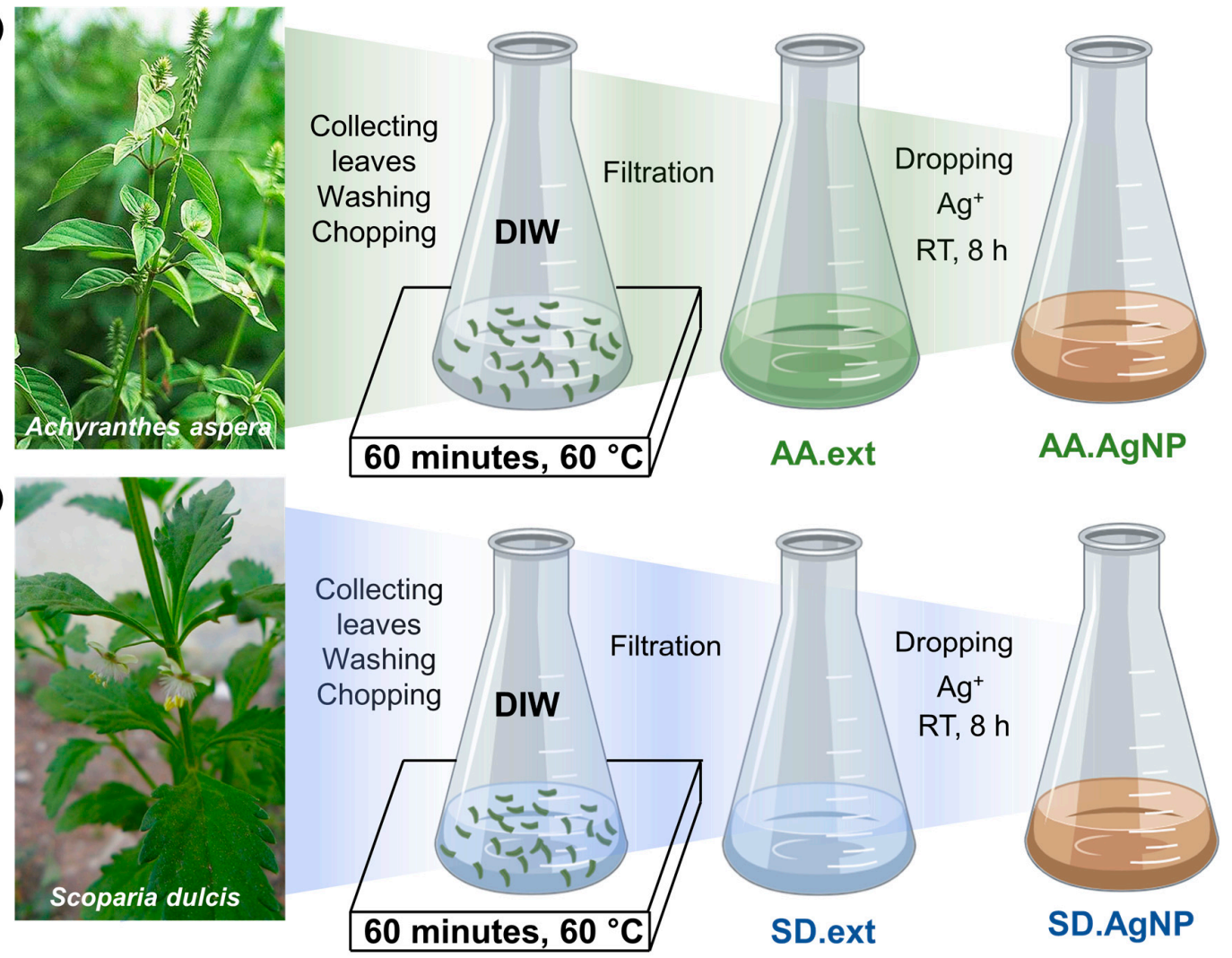

Figure 1. The brief description of the green synthesis procedure of the Achyranthes aspera leaf broth (AA.ext)-mediated silver nanoparticle (AA.AgNP; a) and Scoparia dulcis leaf broth (SD.ext)-mediated silver nanoparticle (SD.AgNP; b).

\subsection{Characterization of AA.AgNP and SD.AgNP Nanoparticles}

The AA.ext, the SD.ext and the reacted solutions fabricating silver nanoparticles after $8 \mathrm{~h}$ were diluted fivefold to scan with a UV-vis spectrophotometer (Shimadzu UV-1800, US). DIW was used to adjust the background in the UV-vis measurement. The wavelength range was set from 350 to $750 \mathrm{~nm}$.

For FTIR spectroscopy, the lyophilized AA.ext, SD.ext, AA.AgNPs and SD.AgNPs were respectively mixed with $\mathrm{KBr}$, and then pressed into pellets. The FTIR (Perkin Elmer, Waltham, Massachusetts, US) was operated in the wavenumber range of $400-4000 \mathrm{~cm}^{-1}$ versus the transmittance mode. 
The elemental compositions of AA.AgNPs and SD.AgNPs were determined by energy dispersive X-ray analysis (EDXA, model H-7593, Horiba, Northampton, UK). Their morphology and dimension were observed by a TEM (JEM-1400, JEOL, Tokyo, Japan) and SEM (FE-SEM S4800, Hitachi, Ibaraki, Japan).

\subsection{Antifungal Activity of AA.AgNP and SD.AgNP Nanoparticles}

The PDA of $39 \mathrm{~g}$ was suspended into DIW of $1 \mathrm{~L}$. Boiling the mixture was done to dissolve completely the PDA powder. Then the PDA solution was sterilized by an autoclave $\left(121^{\circ} \mathrm{C}, 15 \mathrm{~min}\right)$. This pure PDA was poured onto a Petri dish that was named PDA (the control dish). AA.AgNPs were added into PDA solutions at various concentrations of 20 and $40 \mathrm{ppm}$. The dishes were prepared by these media that named were as AA.AgNP20 and AA.AgNP40 for testing the antifungal effect of AA.AgNPs. Similarly, the SD.AgNPs were prepared in PDA solutions at 20 and $40 \mathrm{ppm}$, which were respectively symbolized as SD.AgNP20 and SD.AgNP40 to check the antifungal ability of SD.AgNPs. In addition, two leaf broths were also mixed homogeneously with PDA media to test the antifungal effect of leaf broths only. These dishes were respectively named as AA.ext and SD.ext. The crop pathogen strains including F. oxysporum, A. niger and A. flavus were spotted into the center of each agar dish and incubated at $30^{\circ} \mathrm{C}$. The growing diameter of fungi were observed and measured for each $24 \mathrm{~h}$ during 4 days. Three replicates were done for each mold strain. The results were presented in mean \pm standard deviation. A student's $t$-test was applied to compare the difference between these samples. A $p$-value less than 0.05 would be considered a significant difference. On the contrary, a $p$-value higher than 0.05 would be a non-statistical difference.

\section{Results and Discussion}

\subsection{The Formation of Biosynthesized AgNPs Including AA.AgNPs and SD.AgNPs}

At room temperature, two kinds of silver nanoparticles (AA.AgNPs and SD.AgNPs) were obtained by only one reaction of silver (I) ions with AA.ext and SD.ext. The first observed phenomenon was the color change, the broths had a pale green color and turned to reddish-brown at the stopping point of the reaction. Furthermore, a UV-vis spectrophotometer was utilized to scan two extract solutions and two reacted mixtures containing AA.AgNPs and SD.AgNPs. The UV-vis spectra of AA.ext and SD.ext (Figure 2a,b, dash lines) did not show any peak in the wavelength region of 350-750 nm. Differently, the UV-vis spectra of AA.AgNP and SD.AgNP solution (Figure 2a,b, solid lines) had a peak centered at $433 \mathrm{~nm}$. For both AA.AgNPs and SD.AgNPs, the peak height was increased as a function of reaction time, and was almost saturated after $8 \mathrm{~h}$ of reaction. This peak was caused by the absorption of the plasmonic silver nanoparticles, so the formation of AgNPs was confirmed. However, the AA.AgNPs exhibited a broadened peak, while the SD.AgNPs had a sharp one. The surface plasmon resonance of silver nanoparticles depended on the free electrons interacting with the electromagnetic radiation (light) [32]. Thus, the difference in dimension and morphology or silver nanoparticles could affect on the collective excitation oscillations that might lead to the different absorption peaks. Besides, AA.AgNPs and SD.AgNPs were formed from two different extracts containing the variously reducing amounts, which could lead to creating different concentrations of silver nanoparticles. The broadened peak of AA.AgNPs indicated a higher concentration.

Considering the reaction mechanism, the phytochemicals of A. aspera and S. dulcis played the role of reducing agents, and the silver (I) ions were the oxidizing ones. Due to the abundant compositions in each extract, it might have a lot of oxidation-reduction reactions taking place. However, the phytoconstituents in each plant have not been completely investigated yet, missing understanding would remain. With an attempt connecting all the reported studies, we could comprehend some reactions in detail. A. aspera was reported to have saponins, gallic acid, quercetin, kaempferol, rutin, etc. [18]. Saponins possess the properties of the surfactant having both a hydrophilic and hydrophobic portion. The hydrophilic part is comprised of glycosides, which can reduce silver (I) ions into 
AgNPs [33]. Gallic acid possessing three hydroxyl groups on phenyl rings can cleavage the hydrogen ion and give one electron to silver (I) to become an oxidized gallic acid, and silver (I) ions become AgNPs [34]. In the case of quercetin, kaempferol and rutin, they also have the hydroxyl groups of phenyl ring, and can act as an oxidant with a similar mechanism of gallic acid [35-38]. S. dulcis was found to contain flavonoid, alkaloid and tannin [24] which also possess the hydroxyl groups performing the role of the reductant. Besides, S. dulcis has carbohydrates and glycosides [24], which are well-known as a reductant.
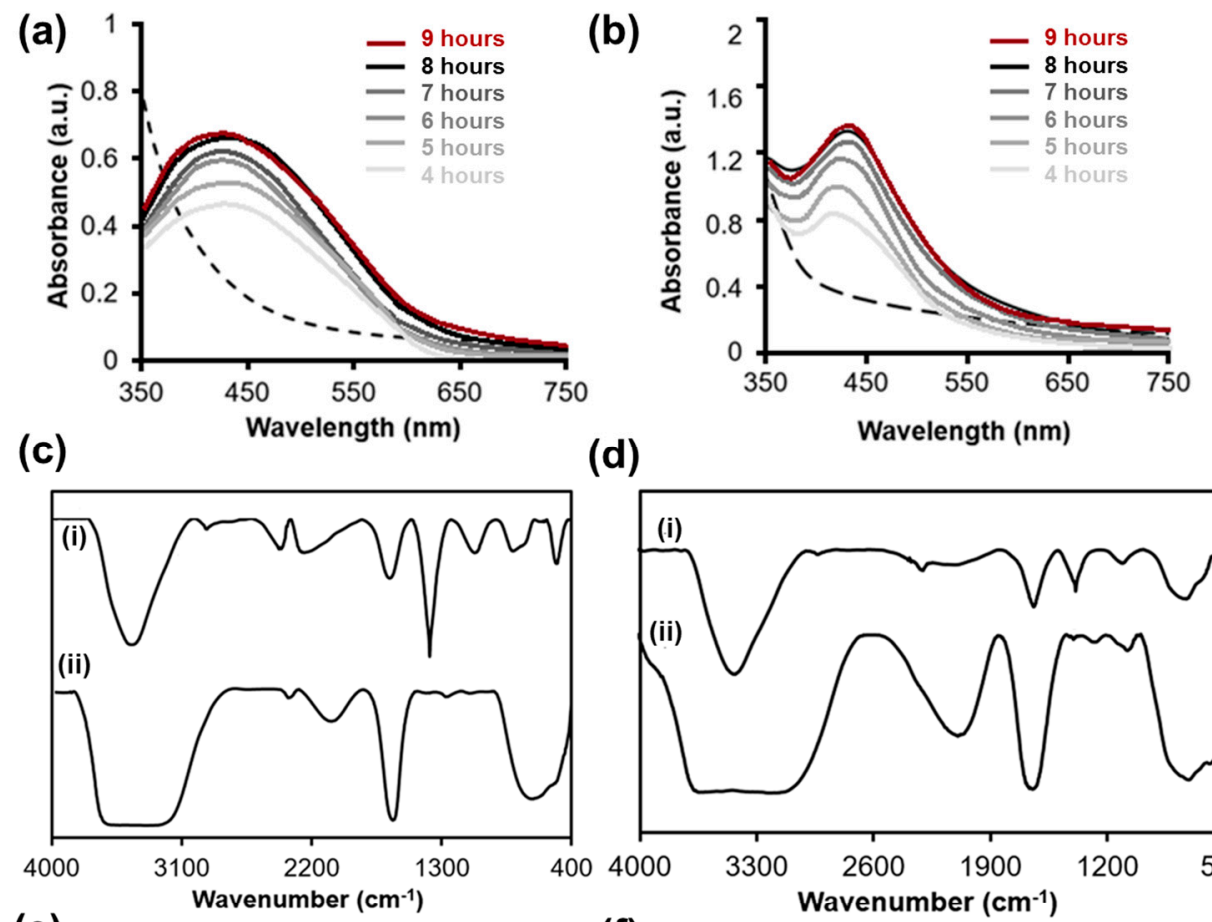

(d)
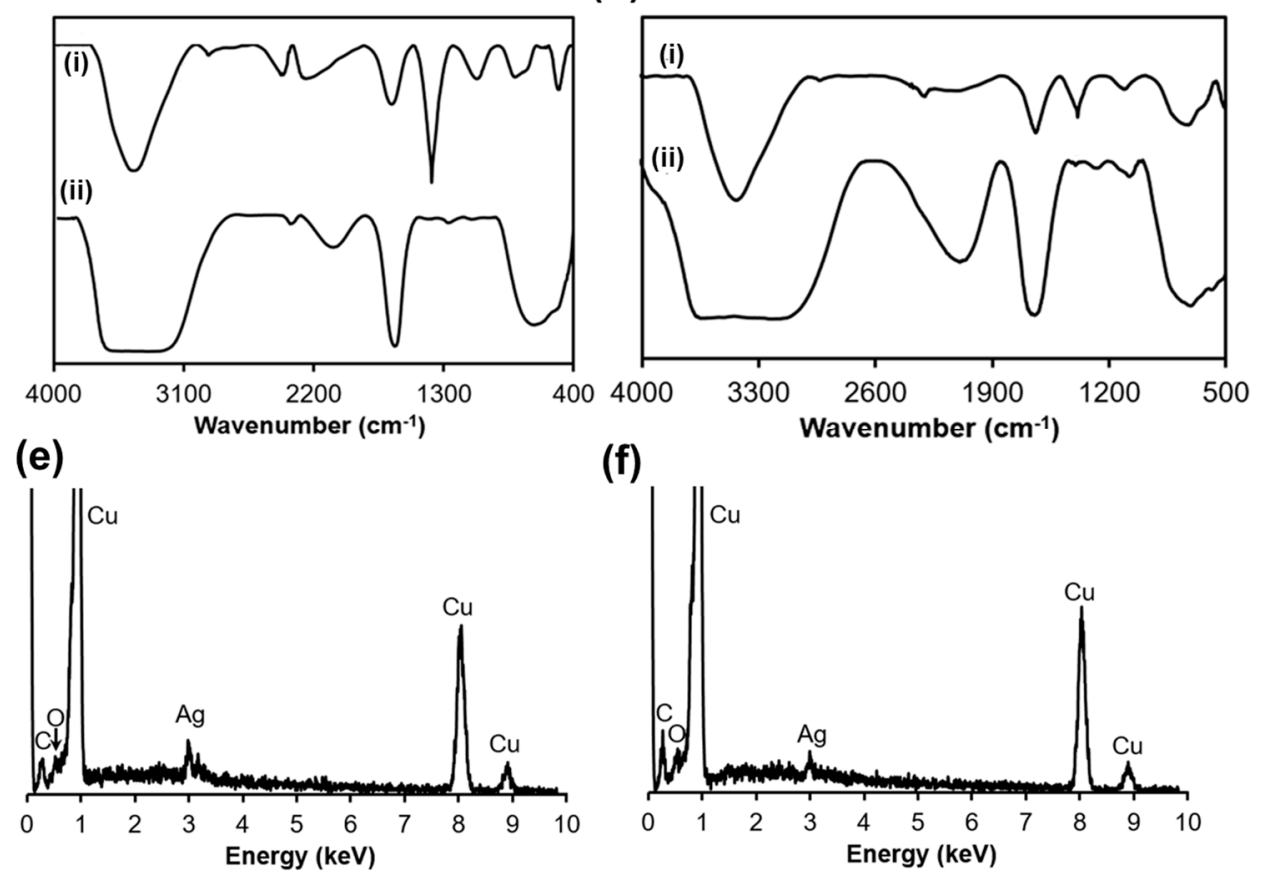

(f)

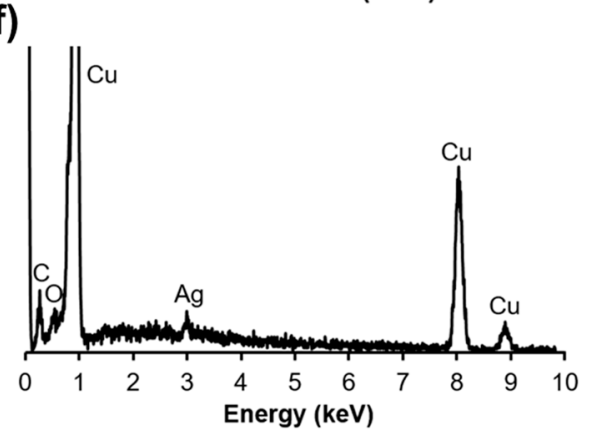

Figure 2. The UV-vis spectra of AA.ext (dash line) and AA.AgNPs at various reaction times (solid line) (a); the UV-vis spectra of SD.ext (dash line) and SD.AgNPs (solid line) (b); the FTIR spectra of AA.AgNPs (i) and AA.ext (ii) (c); the FTIR spectra of SD.AgNPs (i) and SD.ext (ii) (d); the EDXA spectra of AA.AgNPs (e) and SD.AgNPs (f).

On the other hand, these phytoconstituents have a chelating ability with Ag due to containing oxygen of hydroxyl and carboxylic acid groups. It is a beneficial factor to stabilize the biosynthesized silver nanoparticles. Thus, FTIR spectroscopy was performed to explore the functional groups surrounding nanoparticle surfaces. Figure $2 c$ described the FTIR spectra of AA.AgNPs (i) and AA.ext (ii). There was the pointed trough of $3461 \mathrm{~cm}^{-1}$ assigned to the $\mathrm{O}-\mathrm{H}$ stretching band. The O-H groups modified on AA.AgNPs exhibited a sharper trough than O-H of phytoconstituents in the AA.ext extract. It could be explained by the H-bonding in AA.AgNPs being weaker than that in the AA.ext extract. The small trough at $2926 \mathrm{~cm}^{-1}$ and the medium one at $1662 \mathrm{~cm}^{-1}, 1384 \mathrm{~cm}^{-1}$ and $1070 \mathrm{~cm}^{-1}$ were caused by the $\mathrm{C}-\mathrm{H}$ stretching, $\mathrm{C}=\mathrm{O}$ stretching, $\mathrm{O}-\mathrm{H}$ bending and $\mathrm{C}-\mathrm{O}$ stretching vibrations. The 
medium troughs in the region of $840-790 \mathrm{~cm}^{-1}$ were assigned to $C=C$ bending vibration. In the FTIR spectrum of AA.ext (Figure 2c(ii)), all of the troughs of $\mathrm{O}-\mathrm{H}, \mathrm{C}=\mathrm{O}$ and $\mathrm{C}-\mathrm{O}$ groups similarly appeared. Figure $2 \mathrm{~d}(\mathrm{i})$ and $2 \mathrm{~d}$ (ii) describing the FTIR spectra of SD.AgNPs and SD.ext were also observed in the presence of $\mathrm{O}-\mathrm{H}, \mathrm{C}=\mathrm{O}$ and $\mathrm{C}-\mathrm{O}$ groups. Through FTIR results, two biosynthesized silver nanoparticles of AA.AgNPs and SD.AgNPs were proven to be modified by the similar functional groups. However, they were not completely the same because those FTIR spectra having a different fingerprint region $\left(1500-500 \mathrm{~cm}^{-1}\right)$.

The EDXA spectra of AA.AgNPs and SD.AgNPs (Figure 2e,f) showed the signal of $3 \mathrm{keV}$ indicating the silver presence [39]. The signals of $0.28 \mathrm{keV}$ and $0.52 \mathrm{keV}$ were assigned to the carbon and oxygen elements. Besides, the $\mathrm{Cu}$ peaks were observed strongly, which were a strange element in this system. The $\mathrm{Cu}$ contamination came from the sample preparation grid. As a result, AA.AgNPs and SD.AgNPs were demonstrated as the silver nanoparticles covered with organic components composed of carbon and oxygen. These elemental compositions were consisted to FTIR results showing $\mathrm{C}=\mathrm{O}, \mathrm{C}-\mathrm{H}$ and $\mathrm{O}-\mathrm{H}$ bonding vibrations. According to these obtained results in the relation with reported phytoconstituents of A. Aspera and S. dulcis, it was suggested that the silver of AA.AgNPs and SD.AgNPs interacted with the oxygen of phytoconstituents to form the organic shells for stabilizing silver nanoparticles (Figure 3). To confirm this opinion, the SD.AgNP and AA.AgNP solution were stored at $4{ }^{\circ} \mathrm{C}$ in a brown bottle for 15 days. Their UV-vis spectra were measured every 3 days (Figure S1). The 433-nm peaks of AA.AgNPs and SD.AgNPs were not changed about the position, shape and height after a 15-day storage. These results implied that the AA.AgNPs and SD.AgNPs had similar properties of shape and size with their initial form.
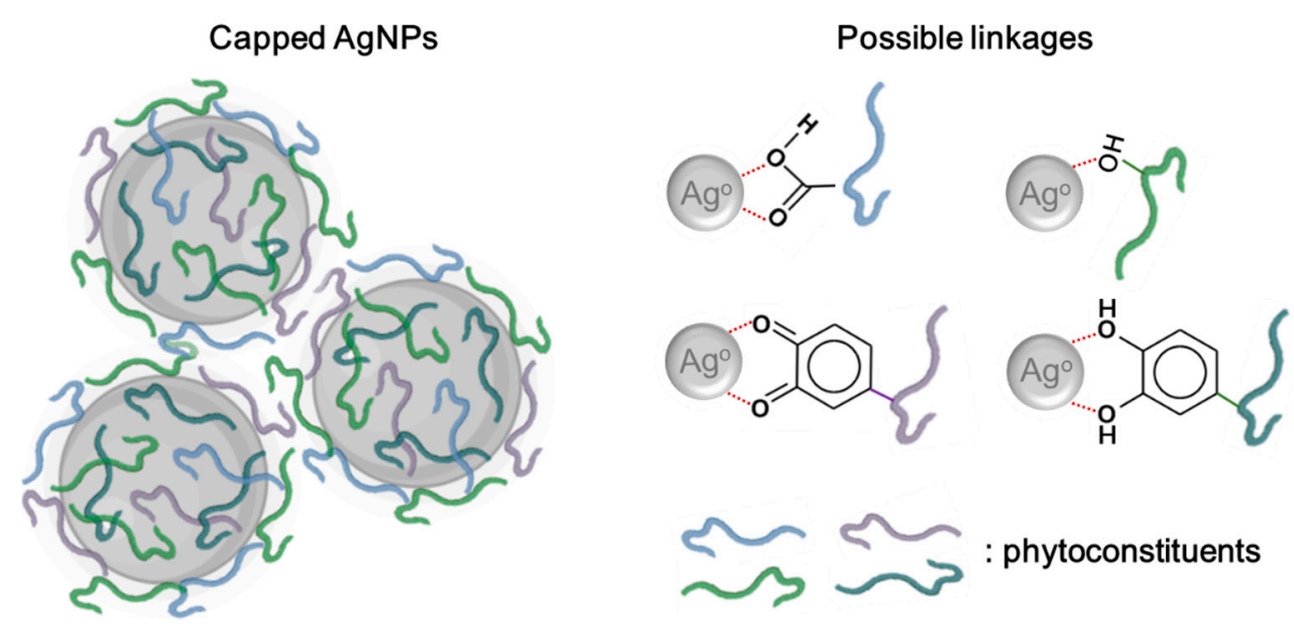

Figure 3. Schematic illustration of biosynthesized silver nanoparticles (AA.AgNPs and SD.AgNPs) capped by phytoconstituents through carboxyl, hydroxyl, quinone and catechol groups and silver interactions.

\subsection{The Morphology and Dimension of AA.AgNPs and SD.AgNPS}

The TEM micrographs of AA.AgNPs and SD.AgNPs were shown in Figure 4a,b. The AA.AgNPs were observed in clusters and almost oval shape (Figure 4a). The size of AA.AgNPs was estimated using image software that was in the range of 8-52 nm and showed the average diameter of $30.5 \pm 9.4 \mathrm{~nm}$. In the earlier study of Elumalai et al. [40], the sizes of A. aspera leaf broth-mediated AgNPs were from 7 to $14 \mathrm{~nm}$, and their morphology were mostly scattered and polydispersed. These different dimensions and morphologies of silver nanoparticles biosynthesized from the $A$. aspera leaf broth were recognized by the geographical location of $A$. aspera. The plants and their phytoconstituent amounts/compositions were effected by weather and soil, $A$. aspera leaf collected from India [40] with different reducing power formed the silver nanoparticles that were not the same as AA.AgNPs. The other reason might be because of the various extracting times leading to varying reductant concentrations. Then, we utilized 
another plant of $S$. dulcis to see how the difference was between two kinds of silver nanoparticles. The SD.AgNPs (Figure $4 \mathrm{~b}$ ) were smaller than AA.AgNPs. In fact, the estimated size of SD.AgNPs was in the scale of $5-45 \mathrm{~nm}$, and their average diameter was $23.1 \pm 9.2 \mathrm{~nm}$. About the morphology, many small SD.AgNPs were nearly of spherical shapes, some bigger SD.AgNPs were of various shapes including oval, quadrilateral, hexagonal and triangle morphology. Additionally, another similar study in India reported that the S. dulcis L. leaf extract assisted in forming the AgNPs in the range of 3-18 nm [41]. Again, the same plants from different countries might be different in phytoconstituent compositions and the amounts that led to the formation of AgNPs of different sizes and shapes.

To observe the nanoparticle surfaces, the SEM microscopy was applied, SEM micrographs of the AA.AgNPs and SD.AgNPs were shown in Figure 4c,d. Most of the nanoparticle surfaces were smooth, the agglomeration could be seen but were still less than the 100-nm clusters. So, using the AA.ext extract, we could obtain the AA.AgNPs distributed in the wide size range and there was more agglomeration than when using the SD.ext extract. These size and morphology results of AA.AgNPs and SD.AgNPs agreed with their UV-vis spectra (Figure 2a,b, solid line). AA.AgNPs had a larger diameter and more clusters than SD.AgNPs, the UV-vis spectrum of AA.AgNPs was broadened more than that of SD.AgNPs. Indeed, these results agreed with Paramelle et al.'s study [42]. The smaller nanoparticles exhibit an absorption, the larger sphere shows increased scattering and shifted to a longer wavelength or broader peak [43]. In the case of the situation where the nanoparticles aggregate, the electrons on the surface of adjacent nanoparticles can delocalize and be shared together, which leads to the decrease of surface plasmon resonance energies. As a result, the UV-vis peak is also scattered or shifted to a longer wavelength.

(a)

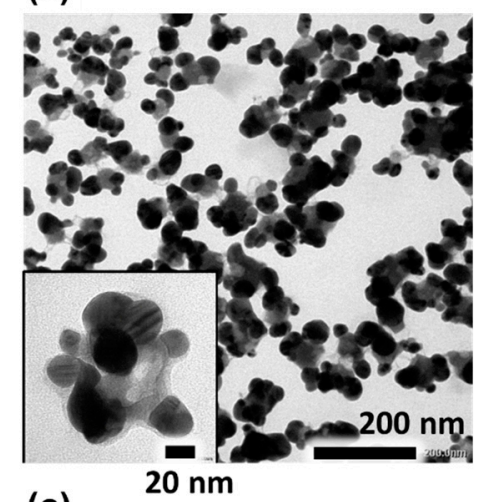

(c)

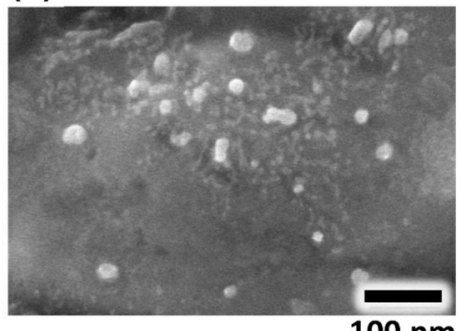

(b)

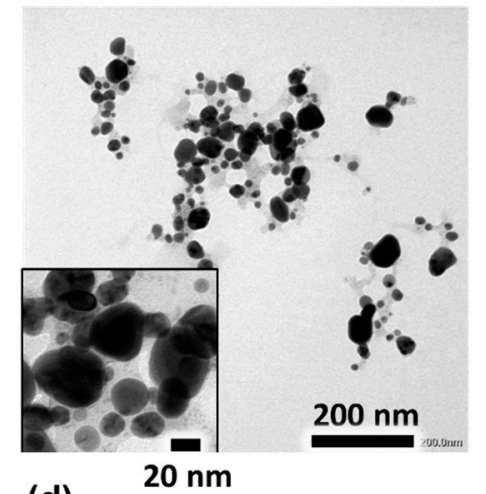

(d)

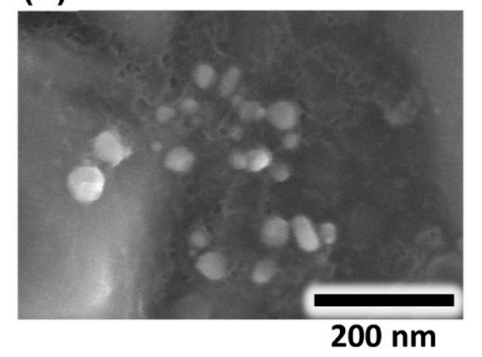

Figure 4. TEM images of AA.AgNPs (a) and SD.AgNPs (b) with the magnification of their photos in the left corner and the SEM micrographs of AA.AgNPs (c) and SD.AgNPs (d).

\subsection{Antifungal Tests}

In order to observe the antifungal effects of AA.ext, SD.ext, AA.AgNPs and SD.AgNPs, three fungal strains including A. niger, A. flavus and F. oxysporum were cultured on the PDA dishes containing those components. Figure 5 showed that three of these fungi on PDA dishes (control sample) were compacted and spread fully on the surface after $96 \mathrm{~h}$. The proliferation of A. niger, A. flavus and F. oxysporum on AA.ext and SD.ext dishes was similar to the control. However, the mycelium zone 
was reduced on AA.AgNP20, AA.AgNP40, SD.AgNP20 and SD.AgNP40, which demonstrated the inhibitory ability of two biosynthesized silver nanoparticles.

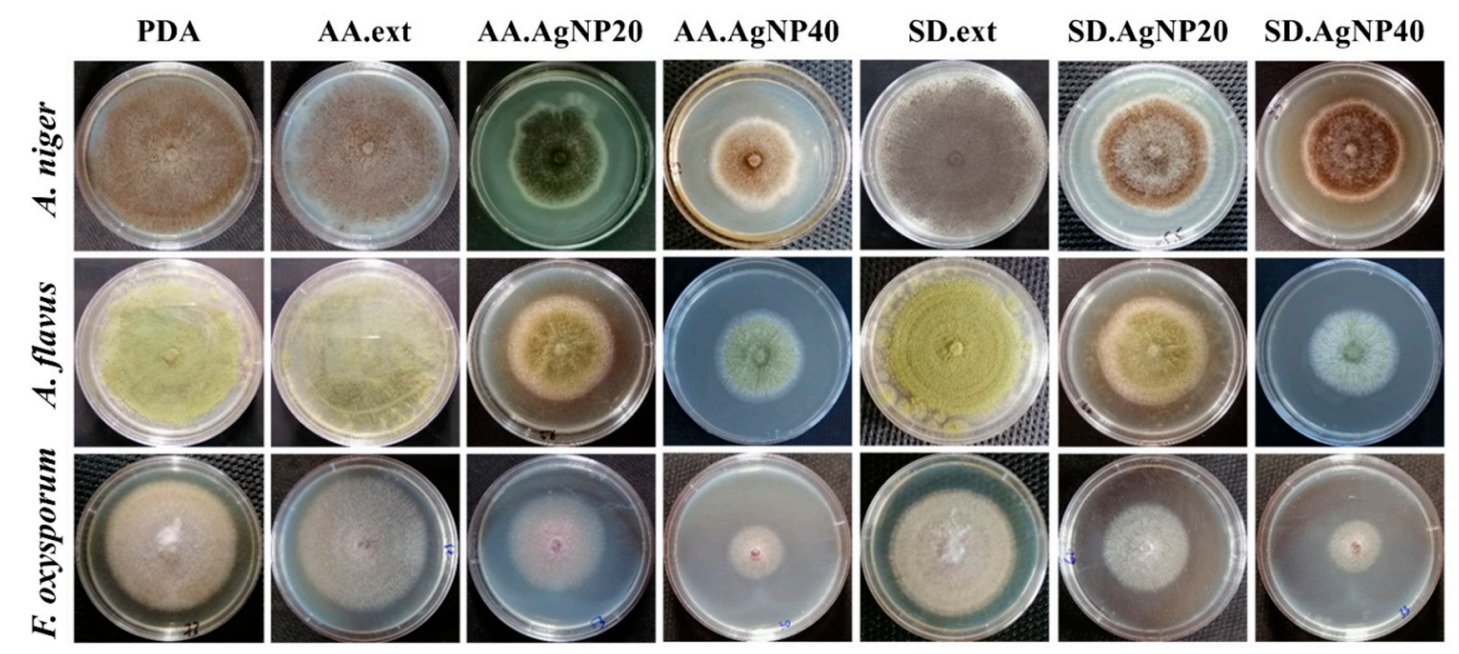

Figure 5. The images of growing zone of Aspergillus niger, Aspergillus flavus and Fusarium oxysporum on various agar dishes including the potato dextrose agar (PDA) dishes (control sample), the PDA dishes containing AA.ext and SD.ext, the PDA dishes containing 20 ppm AA.AgNPs and SD.AgNPs after $96 \mathrm{~h}$ of inoculation.

Quantitatively, the mycelium diameters of each fungal strain on various media were followed every $24 \mathrm{~h}$ until $96 \mathrm{~h}$ when the PDA dishes were full of fungi. In Figure 6a, A. niger created the mycelium circle of $20.7 \mathrm{~mm}$ on PDA, $19.8 \mathrm{~mm}$ on AA.ext and $20.3 \mathrm{~mm}$ on SD.ext after $24 \mathrm{~h}$ of inoculation. Three values were equivalent to each other, which indicated that $A$. niger was not inhibited by neither AA.ext nor SD.ext. After every $24 \mathrm{~h}$, the $A$. niger circles on each dish were larger, which showed the normal proliferation rate. The $A$. niger zones on AA.AgNP20 and AA.AgNP40 were 15.5 and $12.5 \mathrm{~mm}$ after 24 $\mathrm{h}$ of incubation. These values were less than that of PDA dishes. The phenomenon was taken place similarly on SD.AgNP20 and SD.AgNP40, the A. niger diameters were 13.3 and $11.0 \mathrm{~mm}$. These smaller mycelium circles indicated the inhibitory effect of AA.AgNPs and SD.AgNPs against $A$. niger at the concentration of 20 and $40 \mathrm{ppm}$. The high concentration could inhibit more highly the fungal growth. However, 20 and $40 \mathrm{ppm}$ were not enough to kill all fungi, just to reduce the proliferation, thus $A$. niger was slowly spread out until $96 \mathrm{~h}$. Indeed, it was reported that $20 \mathrm{ppm}$ of Mesosilver ${ }^{\circledR}$ (Purest Colloids Inc., Westampton, NJ, USA) could only reduce approximately $25 \%$ of mycelium growth, while $75 \mathrm{ppm}$ inhibited completely the A. niger growth [44]. In the study of Vertelove et al., also testing with $A$. niger, $5 \mathrm{ppm}$ was just the minimal inhibition concentration of silver nanoparticles stabilized by Myramistin ${ }^{\circledR}$ [45]. So, the antifungal capacity of AA.AgNPs and SD.AgNPs against $A$. niger could be comparable with the Mesosilver products and others.

Figure $6 \mathrm{~b}$ showed the mycelium diameters of A. flavus on various media. On PDA, AA.ext and SD.ext, A. flavus were spread out to $24.5,24.1$ and $23.8 \mathrm{~mm}$ after $24 \mathrm{~h}$ of incubation; 44.6, 44.7 and $44.2 \mathrm{~mm}$ after $48 \mathrm{~h} ; 65.3,64.8$ and $64.3 \mathrm{~mm}$ after $72 \mathrm{~h}$ and 89.8, 88.8 and $89.5 \mathrm{~mm}$ after $96 \mathrm{~h}$. These results implied that $A$. flavus were not inhibited by AA.ext and SD.ext extracts and proliferated with the comparable rate to control (PDA). In the case of the presence of AA.AgNP20 and AA.AgNP40, A. flavus' growth was slowed down. In fact, the A. flavus diameters on AA.AgNP20 and AA.AgNP40 dishes were 19.5 and $15.5 \mathrm{~mm}$ after $24 \mathrm{~h}, 31.7$ and $23.8 \mathrm{~mm}$ after $48 \mathrm{~h}, 48.1$ and $39.8 \mathrm{~mm}$ after $72 \mathrm{~h}$ and 66.6 and $53.4 \mathrm{~mm}$ after $96 \mathrm{~h}$. If using the higher silver nanoparticle concentrations, the $A$. flavus diameter was reduced significantly. Changing to other silver nanoparticles of SD.AgNP20 and SD.AgNP40, the A. flavus diameters on dishes were 19.3 and $15.2 \mathrm{~mm}$ after $24 \mathrm{~h}, 30.3$ and $23.3 \mathrm{~mm}$ after $48 \mathrm{~h}, 46.3$ and $36.8 \mathrm{~mm}$ after $72 \mathrm{~h}$ and 65.0 and $52.5 \mathrm{~mm}$ after $96 \mathrm{~h}$. So, two kinds of silver nanoparticles all demonstrated high antifungal activity against $A$. flavus. Looking at the antifungal activity of other 
silver nanoparticle products against $A$. flavus [46,47], these results of AA.AgNPs and SD.AgNPs were considered as a similar effect. The silver nanoparticles of the American company MTI (Aladdin, WY, USA) could inhibit the fungus A. flavus effectively at the concentration of 150-200 ppm [46]. Differently, silver nanoparticles Biopure-AgNP (NanoComposix, San Diego, CA, USA) could show their successful inhibition at $80 \mathrm{ppm}$ [47].

\section{(a)}

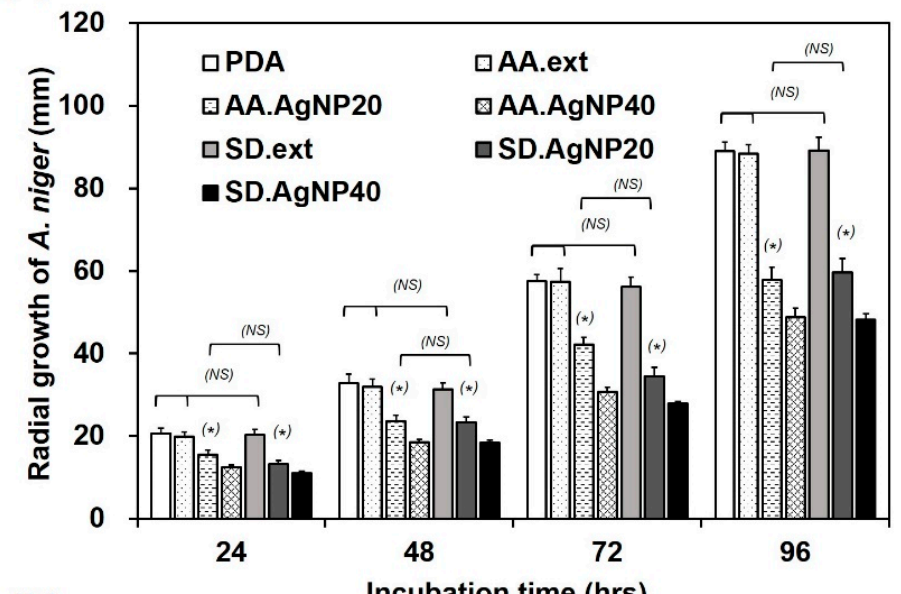

(b)

Incubation time (hrs)
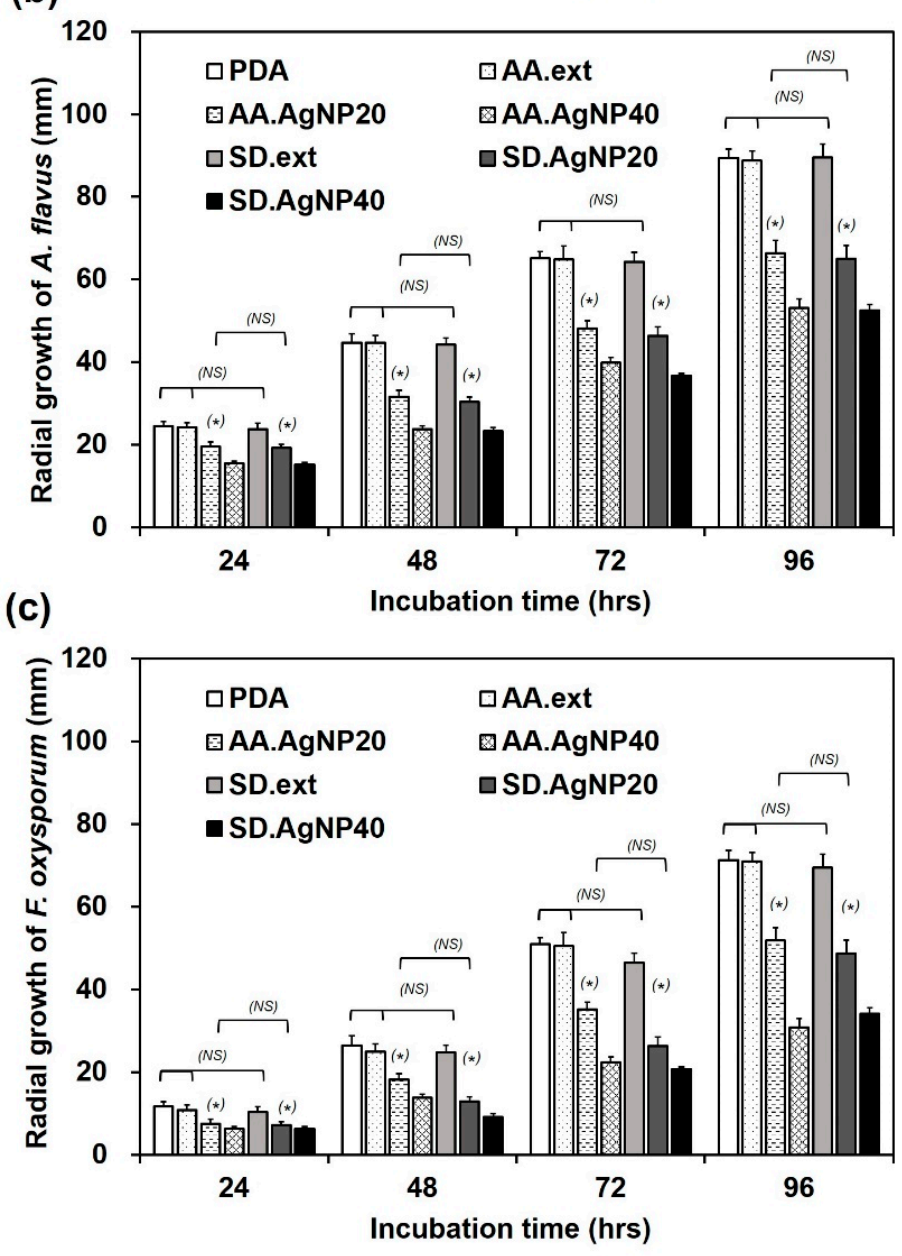

Figure 6. The growing diameters of Aspergillus niger (a), Aspergillus flavus (b) and Fusarium oxysporum (c) on various agar dishes including the PDA dishes (control sample), the PDA dishes containing AA.ext and SD.ext and the PDA dishes containing 20 ppm AA.AgNPs and SD.AgNPs. 
Figure $6 \mathrm{c}$ described the fungal diameters of F. oxysporum, this strain was also not impacted by AA.ext and SD.ext, which was proven by the same diameter growth between PDA and two extract dishes. When F. oxysporum was treated with AA.AgNP20, AA.AgNP40, SD.AgNP20 and SD.AgNP40, its mycelium diameters were also decreased as a function of the silver nanoparticle concentration. In the study of Aleksandrowicz-Trzcińska et al., F. oxysporum was treated with commercial AgNPs (Nano-Kolid Sp. z o. o, Warszawa, Poland), AgNPs showed the decrease of the mycelium zone significantly at 15, 25 and 35 ppm [48]. Through this value, the AA.AgNPs and SD.AgNPs could be said that they achieved a similar antifungal capacity with commercial AgNPs. When compared to two aforementioned strains, F. oxysporum exhibited a weaker ability in contact with silver nanoparticles. After $96 \mathrm{~h}$, F. oxysporum diameters were 51.8 and $30.3 \mathrm{~mm}$ when contacting AA.AgNP20 and AA.AgNP40 and 48.7 and $34.2 \mathrm{~mm}$ when contacting SD.AgNP20 and SD.AgNP40. These values were significantly less than that of A. flavus and A. niger on similar media and conditions. Altogether, the biosynthesized silver nanoparticles of either AA.AgNPs or SD.AgNPs revealed their effectively antifungal ability against three strains of $A$. flavus, F. oxysporum and A. niger.

In addition, the strong points of plant extract-mediated silver nanoparticles were confirmed to inherently possess an antibacterial activity and superior biocompatibility [49] due to nontoxic and naturally reducing and stabilizing plant molecules. However, the overall biocompatibility of AgNPs was mainly governed by concentrations. Using the rat aortic ring model, green AgNPs of 50-300 ppm were demonstrated as an antiangiogenesis agents for biomedical applications [50]. Amin et al. utilized green AgNPs to treat the infected rats through oral administration at the AgNPs concentration of 16 ppm [51]. There were no side effects caused by green AgNPs. Taken together, the biosynthesized AgNPs were considered as biocompatible agents at working doses.

\section{Conclusions}

In conclusion, an ecofriendly synthesis of AgNPs using A. aspera and S. dulcis leaf extract was successfully performed. This method provided an alternative approach for silver nanoparticle fabrication to gradually replace traditional methods. A. aspera and S. dulcis leaf extract had the ability to reduce silver (I) ions into AA.AgNPs and SD.AgNPs. These final silver nanoparticle products were obtained and characterized by UV-Vis spectroscopy, FT-IR, EDXA analysis, TEM and SEM micrographs. AA.AgNPs exhibited the peak centered at $433 \mathrm{~nm}$ and broadened more than SD.AgNPs in UV-vis spectra. Both AA.AgNPs and SD.AgNPs were modified by phytoconstituents containing C=C, C-O and $\mathrm{O}-\mathrm{H}$ bonds, but each specific surface was different due to different reducing/stabilizing sources. AA.AgNPs were bigger and had more agglomeration than SD.AgNPs, but two types of these silver nanoparticles were less than $55 \mathrm{~nm}$. In addition, the synthesized AgNPs showed high antifungal activity against three fungal strains including A. flavus, A. niger and F. oxysporum. Especially, the F. oxysporum had the most strongly reduced growth rate when contacting AA.AgNPs and SD.AgNPs. By the one-pot reaction based on the green chemistry approach, two new silver nanoparticles were fabricated and had promising scalability for various applications, especially in medical and agricultural fields as an alternative fungicide.

Supplementary Materials: The following are available online at http://www.mdpi.com/2076-3417/10/7/2505/s1, Figure S1: The UV-vis spectra of AA.AgNPs (a) and SD.AgNPs (b) stored at $4{ }^{\circ} \mathrm{C}$ in a brown bottle after various time intervals.

Author Contributions: Author Contributions: Conceptualization, N.T.T.L.; methodology, N.T.T.L., H.N.T.N. and T.T.H.T.; formal analysis, N.T.T.L. and D.Y.P.N.; data curation, N.T.T.L., N.H.N., Y.C.C., D.Y.P.N. and C.Q.N.; writing - review and editing, T.T.H.T.; supervision, D.H.N., Y.C.C. and T.T.H.T.; project administration, D.H.N.; funding acquisition, D.H.N. All authors have read and agreed to the published version of the manuscript.

Acknowledgments: This research was supported by the Domestic Master/PhD Scholarship Program of Vingroup Innovation Foundation (Grant number: VINIF.2019.TS.72).

Conflicts of Interest: The authors declare no conflict of interest. 


\section{References}

1. Hendrickson, J.A.; Hu, C.; Aitken, S.L.; Beyda, N. Antifungal Resistance: A Concerning Trend for the Present and Future. Curr. Infect Dis. Rep. 2019, 21, 47. [CrossRef] [PubMed]

2. Sharma, P.; Sharma, A.; Sharma, M.; Bhalla, N.; Estrela, P.; Jain, A.; Thakur, P.; Thakur, A. Nanomaterial Fungicides: In Vitro and In Vivo Antimycotic Activity of Cobalt and Nickel Nanoferrites on Phytopathogenic Fungi. Glob. Chall. 2017, 1, 1700041. [CrossRef] [PubMed]

3. Thanh, V.M.; Bui, L.M.; Bach, L.G.; Nguyen, N.T.; Thi, H.L.; Hoang Thi, T.T. Origanum majorana L. Essential Oil-Associated Polymeric Nano Dendrimer for Antifungal Activity against Phytophthora infestans. Materials 2019, 12, 1446. [CrossRef] [PubMed]

4. Hao, Y.; Cao, X.; Ma, C.; Zhang, Z.; Zhao, N.; Ali, A.; Hou, T.; Xiang, Z.; Zhuang, J.; Wu, S.; et al. Potential Applications and Antifungal Activities of Engineered Nanomaterials against Gray Mold Disease Agent Botrytis cinerea on Rose Petals. Front. Plant Sci. 2017, 8. [CrossRef]

5. Grace, J.L.; Huang, J.X.; Cheah, S.-E.; Truong, N.P.; Cooper, M.A.; Li, J.; Davis, T.P.; Quinn, J.F.; Velkov, T.; Whittaker, M.R. Antibacterial low molecular weight cationic polymers: Dissecting the contribution of hydrophobicity, chain length and charge to activity. RSC Adv. 2016, 6, 15469-15477. [CrossRef]

6. Grace, J.L.; Elliott, A.G.; Huang, J.X.; Schneider, E.K.; Truong, N.P.; Cooper, M.A.; Li, J.; Davis, T.P.; Quinn, J.F.; Velkov, T.; et al. Cationic acrylate oligomers comprising amino acid mimic moieties demonstrate improved antibacterial killing efficiency. J. Mater. Chem. B 2017, 5, 531-536. [CrossRef]

7. Barbon, S.M.; Truong, N.P.; Elliott, A.G.; Cooper, M.A.; Davis, T.P.; Whittaker, M.R.; Hawker, C.J.; Anastasaki, A. Elucidating the effect of sequence and degree of polymerization on antimicrobial properties for block copolymers. Polym. Chem. 2020, 11, 84-90. [CrossRef]

8. Hoang Thi, T.T.; Lee, Y.; Le Thi, P.; Park, K.D. Nitric oxide-releasing injectable hydrogels with high antibacterial activity through in situ formation of peroxynitrite. Acta Biomater. 2018, 67, 66-78. [CrossRef]

9. $\quad$ Brauer, V.S.; Rezende, C.P.; Pessoni, A.M.; De Paula, R.G.; Rangappa, K.S.; Nayaka, S.C.; Gupta, V.K.; Almeida, F. Antifungal Agents in Agriculture: Friends and Foes of Public Health. Biomolecules 2019, 9, 521. [CrossRef]

10. Baker, S.; Nagendra Prasad, M.N.; Chouhan, R.S.; Mohan Kumar, K.; Satish, S. Development of bioconjugated nano-molecules against targeted microbial pathogens for enhanced bactericidal activity. Mater. Chem. Phys. 2020, 242, 122292. [CrossRef]

11. Hebbalalu, D.; Lalley, J.; Nadagouda, M.N.; Varma, R.S. Greener Techniques for the Synthesis of Silver Nanoparticles Using Plant Extracts, Enzymes, Bacteria, Biodegradable Polymers, and Microwaves. ACS Sustain. Chem. Eng. 2013, 1, 703-712. [CrossRef]

12. Oh, J.-W.; Chun, S.C.; Chandrasekaran, M. Preparation and In Vitro Characterization of Chitosan Nanoparticles and Their Broad-Spectrum Antifungal Action Compared to Antibacterial Activities against Phytopathogens of Tomato. Agronomy 2019, 9, 21. [CrossRef]

13. Hassan, S.A.; Hanif, E.; Khan, U.H.; Tanoli, A.K. Antifungal activity of silver nanoparticles from Aspergillus niger. Pak. J. Pharm. Sci. 2019, 32, 1163-1166. [PubMed]

14. Iravani, S. Bacteria in Nanoparticle Synthesis: Current Status and Future Prospects. Int. Sch. Res. Not. 2014, 2014, 359316. [CrossRef] [PubMed]

15. Sharma, A.; Tripathi, P.; Kumar, S. One-pot synthesis of silver nanocomposites from Achyranthes aspera: An eco-friendly larvicide against Aedes aegypti L. Asian Pac. J. Trop. Biomed. 2020, 10, 54-64. [CrossRef]

16. Nguyen, D.H.; Lee, J.S.; Park, K.D.; Ching, Y.C.; Nguyen, X.T.; Phan, V.H.G.; Hoang Thi, T.T. Green Silver Nanoparticles Formed by Phyllanthus urinaria, Pouzolzia zeylanica, and Scoparia dulcis Leaf Extracts and the Antifungal Activity. Nanomaterials 2020, 10, 542. [CrossRef] [PubMed]

17. Singh, J.; Dutta, T.; Kim, K.H.; Rawat, M.; Samddar, P.; Kumar, P. 'Green' synthesis of metals and their oxide nanoparticles: Applications for environmental remediation. J. nanobiotechnol. 2018, 16, 84. [CrossRef]

18. Viswanatha, G.L.; Venkataranganna, M.V.; Prasad, N.B.L.; Godavarthi, A. Achyranthes aspera Attenuates epilepsy in experimental animals: Possible involvement of GABAergic mechanism. Metab. Brain Dis. 2017, 32, 867-879. [CrossRef]

19. Khandagle, A.J.; Tare, V.S.; Raut, K.D.; Morey, R.A. Bioactivity of essential oils of Zingiber officinalis and Achyranthes aspera against mosquitoes. Parasitol. Res. 2011, 109, 339-343. [CrossRef] 
20. Mali, R.G.; Dhake, A.S. A review on herbal antiasthmatics. Orient. Pharm. Exp. Med. 2011, 11, 77-90. [CrossRef]

21. Edwin, S.; Jarald, E.E.; Deb, L.; Jain, A.; Kinger, H.; Dutt, K.R.; Raj, A.A. Wound Healing and Antioxidant Activity ofAchyranthes aspera. Pharm. Biol. 2009, 46, 824-828. [CrossRef]

22. Bhoomika, R.G.; Ramesh, K.G.; Anita, A.M. Phyto-pharmacology of Achyranthes aspera: A Review. Pharmacogn. Rev. 2007, 1, 143.

23. Liu, Q.; Yang, Q.M.; Hu, H.J.; Yang, L.; Yang, Y.B.; Chou, G.X.; Wang, Z.T. Bioactive diterpenoids and flavonoids from the aerial parts of Scoparia dulcis. J. Nat. Prod. 2014, 77, 1594-1600. [CrossRef] [PubMed]

24. Meera, P.; Kavitha, V.; Krishnaja, K.R. Scoparia dulcis: A review on its phytochemical and pharmacological profile. Innoriginal Int. J. Sci. 2017, 4, 18-22.

25. Ahmed, S.; Saifullah; Ahmad, M.; Swami, B.L.; Ikram, S. Green synthesis of silver nanoparticles using Azadirachta indica aqueous leaf extract. J. Radiat. Res. Appl. Sci. 2016, 9, 1-7. [CrossRef]

26. Bar, H.; Bhui, D.K.; Sahoo, G.P.; Sarkar, P.; Pyne, S.; Misra, A. Green synthesis of silver nanoparticles using seed extract of Jatropha curcas. Colloids Surf. A Physicochem. Eng. Asp. 2009, 348, 212-216. [CrossRef]

27. Singh, P.; Pandit, S.; Garnæs, J.; Tunjic, S.; Mokkapati, V.R.; Sultan, A.; Thygesen, A.; Mackevica, A.; Mateiu, R.V.; Daugaard, A.E.; et al. Green synthesis of gold and silver nanoparticles from Cannabis sativa (industrial hemp) and their capacity for biofilm inhibition. Int. J. Nanomed. 2018, 13, 3571-3591. [CrossRef]

28. Doan, P.; Cao, V.; Nguyen, D.H.; Tran, N.Q. Metallic nanoparticles: Potential ecofungicide for controlling growth of plant-pathogenic fungi. J. Chem. Soc. Pak. 2018, 40, 664-675.

29. Ruchi, S. Pathogenecity of Aspergillus niger in plants. Cibtech J. Microbiol. 2012, 1, 47-51.

30. Brown, G.D.; Denning, D.W.; Gow, N.A.; Levitz, S.M.; Netea, M.G.; White, T.C. Hidden killers: Human fungal infections. Sci. Transl. Med. 2012, 4, 165rv113. [CrossRef]

31. Michielse, C.B.; Rep, M. Pathogen profile update: Fusarium oxysporum. Mol. Plant Pathol. 2009, 10, 311-324. [CrossRef]

32. Pineider, F.; Sangregorio, C. Chapter 6-Nanomaterials for Magnetoplasmonics. In Novel Magnetic Nanostructures; Domracheva, N., Caporali, M., Rentschler, E., Eds.; Elsevier: New York, NY, USA, 2018; pp. 191-220. [CrossRef]

33. Gerami, M.; Ramezani, M.; Majlesi, Z. Investigation on some main glycosides content of Stevia rebaudian B. under different concentrations of commercial and synthesized silver nanoparticles. Pharm. Biomed. Res. 2018. [CrossRef]

34. Sutradhar, P.; Saha, M. Size-controlled synthesis of silver nanoparticles using Zizyphus mauritiana fruit extract. Main Group Chem. 2015, 15, 47-55. [CrossRef]

35. Mirgorod, Y.A.; Borodina, V.G.; Borsch, N.A. Investigation of interaction between silver ions and rutin in water by physical methods. Biophysics 2014, 58, 743-747. [CrossRef]

36. Halder, A.; Das, S.; Bera, T.; Mukherjee, A. Rapid synthesis for monodispersed gold nanoparticles in kaempferol and anti-leishmanial efficacy against wild and drug resistant strains. RSC Adv. 2017, 7, 14159-14167. [CrossRef]

37. Jain, S.; Mehata, M.S. Medicinal Plant Leaf Extract and Pure Flavonoid Mediated Green Synthesis of Silver Nanoparticles and their Enhanced Antibacterial Property. Sci. Rep. 2017, 7, 15867. [CrossRef] [PubMed]

38. Chikkanna, M.M.; Neelagund, S.E. Effect of Sheep and Goat Fecal Mediated Synthesis and Characterization of Silver Nanoparticles (AgNPs) and Their Antibacterial Effects. J. Nanofluids 2018, 7, 309-315. [CrossRef]

39. Bar, H.; Bhui, D.K.; Sahoo, G.P.; Sarkar, P.; De, S.P.; Misra, A. Green synthesis of silver nanoparticles using latex of Jatropha curcas. Colloids Surf. A Physicochem. Eng. Asp. 2009, 339, 134-139. [CrossRef]

40. Elumalai, D.; Kaleena, P.K.; Ashok, K.; Suresh, A.; Hemavathi, M. Green synthesis of silver nanoparticle using Achyranthes aspera and its larvicidal activity against three major mosquito vectors. Eng. Agric. Environ. Food 2016, 9, 1-8. [CrossRef]

41. Parvataneni, R. Biogenic synthesis and characterization of silver nanoparticles using aqueous leaf extract of Scoparia dulcis L. and assessment of their antimicrobial property. Drug Chem. Toxicol. 2019, 43, 307-321. [CrossRef]

42. Paramelle, D.; Sadovoy, A.; Gorelik, S.; Free, P.; Hobley, J.; Fernig, D.G. A rapid method to estimate the concentration of citrate capped silver nanoparticles from UV-visible light spectra. Analyst 2014, 139, 4855-4861. [CrossRef] [PubMed] 
43. Nayfeh, E.M. Fundamentals and Applications of Nano Silicon in Plasmonics and Fullerines: Current and Future Trends, Chapter 8: Nanometal. Micro Nano Technol. 2018, 169-203. [CrossRef]

44. Colloidal Silver Study Results: Bacteriology. Available online: https://www.silver-colloids.com/biostudies/ (accessed on 30 March 2020).

45. Marambio-Jones, C.; Hoek, E.M.V. A review of the antibacterial effects of silver nanomaterials and potential implications for human health and the environment. J. Nanopartic. Res. 2010, 12, 1531-1551. [CrossRef]

46. Muna, A.; Halima, Z.H. Antifungal effect of silver nanoparticles (AgNPs) against Aspergillus flavus. EC Microbiol. 2017, 6, 63-66.

47. Villamizar-Gallardo, R.; Cruz, J.F.O.; Ortíz-Rodriguez, O.O. Fungicidal effect of silver nanoparticles on toxigenic fungi in cocoa. Pesquisa Agropecuária Brasileira 2016, 51, 1929-1936. [CrossRef]

48. Marta, A.-T.S.; Adam, S.; Jacek, O.; Stanisław, D. Effects of copper and silver nanoparticles on growth of selected species of pathogenic and wood-decay fungi in vitro. For. Chron. 2018, 94, 109-116.

49. Augustine, R.; Hasan, A. Emerging applications of biocompatible phytosynthesized metal/metal oxide nanoparticles in healthcare. J. Drug Deliv. Sci. Technol. 2020, 56, 101516. [CrossRef]

50. Baharara, J.; Namvar, F.; Ramezani, T.; Hosseini, N.; Mohamad, R. Green Synthesis of Silver Nanoparticles using Achillea biebersteinii Flower Extract and its Anti-Angiogenic Properties in the Rat Aortic Ring Model. Molecules 2014, 19, 4624-4634. [CrossRef]

51. Amin, M.; Hameed, S.; Ali, A.; Anwar, F.; Shahid, S.A.; Shakir, I.; Yaqoob, A.; Hasan, S.; Khan, S.A.; Sajjad Ur, R. Green Synthesis of Silver Nanoparticles: Structural Features and In Vivo and In Vitro Therapeutic Effects against Helicobacter pylori Induced Gastritis. Bioinorg. Chem. Appl. 2014, 2014, 135824. [CrossRef]

(C) 2020 by the authors. Licensee MDPI, Basel, Switzerland. This article is an open access article distributed under the terms and conditions of the Creative Commons Attribution (CC BY) license (http://creativecommons.org/licenses/by/4.0/). 